\title{
Recognize Facial Impression using Artificial Neural Network
}

\author{
Montaser H.B. Ali and Osman M. Elfadil
}

\begin{abstract}
- this paper aims to design an artificial neural network to discover the impression by recognizing the expression of the human face. To achieve this goal, the artificial neural network was analyzed and to create patterns of the database containing a set of images with different expressions. The learning process of the network was also conducted through patterns training. The extent to which patterns of online training were recognized was compared to the true values of expressions. The grid was trained in 200 patterns and the anomalies were removed. Then re-learned the network again and analyzed the network performance by comparing the real expression with the expected expression and outputting the error for the network appearing. Impression recognition in the grid applied a three-layer back propagation model, with an average error of 0.321 . The performance of the artificial neural network in the recognition of impressions was $80 \%$
\end{abstract}

Index Terms-FER, ANN, JAFFE, Facial, Recognition, Expression, Impression, Pattern, Training

\section{INTRODUCTION}

Facial expressions are one of the more important aspects of human communication.Face Expression variations produced by this factor are due to the inherent dynamic nature of the faces and arise in daily life during interactions and conversations introducing distortions regarding the neutral expression. Thus, as each basic facial expression is associated to a finite combination of facial muscles there is the possibility of finding invariants and transformations which might help FR systems. In this sense, although FR research techniques have traditionally considered facial expressions as a distortion that negatively influences recognition ratio, we should also take into account that expressions contain reliable information which is used by humans for identifying subjects. In addition, a face will be easier identified at a given local scene, depending on the emotion currently expressed. Emotional facial expressions by its side, refers to a specific subset of the general human expressions. Facial expressions" representing happiness, sadness, anger, fear, surprise and disgust according of six universal expression basic emotions [1].

A neural network can be thought of as a network of "neurons" organized in layers. The number of types of Artificial Neural Networks (ANNs) and their uses can potentially be very high. Since the first neural model by McCulloch and Pitts there have been developed hundreds of different models considered as ANNs. The differences in them might be the functions, the accepted values, the topology, the learning algorithms, etc [2]. Also there are many hybrid models. Since the function of ANNs is to process information, they are used mainly in fields related to it. An ANN is formed from single units, (artificial neurons or Processing Elements - PE), connected with coefficients (weights), which constitute the neural structure and are organized in layers. The power of neural computations comes from connecting neurons in a network. Each PE has weighted inputs, transfer function and one output. The behavior of a neural network is determined by the transfer functions of its neurons, by the learning rule, and by the architecture itself. The weights are the adjustable parameters and, in that sense, a neural network is a parameterized system. The weighed sum of the inputs constitutes the activation of the neuron. An ANN is typically defined by three types of parameters:

1. The interconnection pattern between the different layers of neurons.

2. The learning process for updating the weights of the weights.

3. The activation function that converts a neuron's weighted input to its output activation [3].

How should the neurons be connected together? If a network is to be of any use, there must be inputs and outputs. However, there also can be hidden neurons that play an internal role in the network. The input, hidden and output neurons need to be connected together. A simple network has a feed-forward structure: signals flow from inputs, forwards through any hidden units, eventually reaching the output units. However, if the network is recurrent (contains connections back from later to earlier neurons) it can be unstable, and has a very complex dynamics. Recurrent networks are very interesting to researchers in neural networks, but so far it is the feedforward structures that have proved most useful in solving real problems [4].

\section{FACE RECOGNITION ALGORITHMS}

Face recognition is the process of identifying or verifying a person from image or a video frame from a video source. There are many algorithms used in face recognition such as appearance based, active appearance, support vector machines, Bayesian model, neural network, texture based and feature based [5].

\section{A. Appearance Based}

Appearance based algorithms use image pixel data as a whole for recognition.

Direct Correlation, Eigen-face and fisher-face belong to this class of algorithms. Direct correlation uses direct comparison of image pixels of two facial images, producing a similarity score. Unlike a direct correlation method that uses facial images in their original image space, Eigen-face and fisher-face algorithms reduce the image to the most discriminating factor and make their comparison between images in a reduced dimension image space [6].

\section{B. Active Appearance}

Active Appearance model algorithms contain statistical information of an image shape and texture variation. Applied principal component analysis to generate statistical model that localized land marks on the training set of 
images.the landmarks are used to learn displacement between a synthesized model parameter and the training images. To match an image, the current residual error of the model is measured against predicating changes to current model parameters leading to a better fit and recognition [5].

\section{Support Vector Machines}

Support Vector Machines use a training set of images to compute the optimal separating hyper plane. Applied this method to face recognition using a binary tree classification, where face images are iteratively classified as belonging to one of the two classes that propagates up a binary tree structure until a final classification decision can be made [7].

\section{Bayesian Model}

The association of prior distribution with unknown is called Bayesian Modeling.

Bayesian Model algorithms show a probabilistic measure of similarity derived from a Bayesian Analysis of the difference between face images. Computing probability functions using the differences of image intensity in two sets of data, leads to a similarity score from Bayes rule, which can then be used for recognition classification [8].

\section{E. Texture Based}

Texture based algorithms extract textual features from face images, by dividing a face into several regions. Local Binary Pattern (LBP) is an example of Texture based algorithms, where weighted LBP features are extracted to generate a feature vector. Two LBP feature vector are matched by applying weighted chi-squared distance measure [9].

\section{F. Feature Based}

These algorithms extract a set of geometrical features and distances from facial images and use these features as the basis of comparison between images. Local Feature Analysis is an example of feature based algorithms [7].

\section{SCHEME OF WORK}

Scheme of work steps to recognize facial impression as following:

\section{A. Learning pattern and generation}

\section{1) Data Acquisitions}

Japanese Female Face Expression (JAFFE) data base used as input data in neural network designed model. JAFFE consist of 216 ten female every once have seven expression, Happy, Sad, Angry, Surprised, Disgusts, Fear and Nervous on each image with a $256 \times 256$ resolution which is represented the actual output code after training data as Fig.1
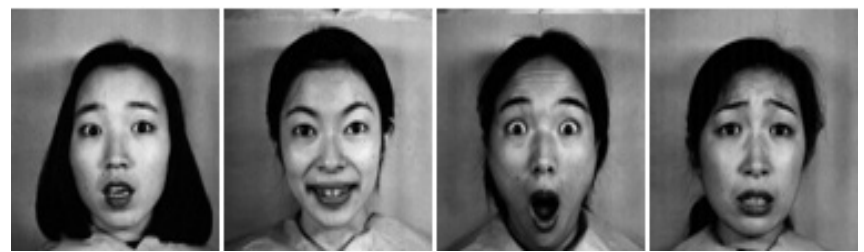

Fig. 1 JAFFE Data Base

2) Data Processing and Pattern Generation
The images expression data base input was resize to $100 \times 100$ by used Microsoft office picture manager and photo image editor converted images format to bitmap (bmp) then converted data base images to ASCII using Erdas 9.2 software after that collected all images.org in excel file and added output code which are pattern file.As in Fig. 2

\begin{tabular}{|c|c|c|c|c|c|c|c|c|c|c|c|c|c|}
\hline 2 & & & & c & & E & & G & & 1 & 1 & K & \\
\hline$\frac{1}{2}$ & 7 & & $\begin{array}{l}77 \\
87\end{array}$ & $\begin{array}{l}87 \\
99\end{array}$ & $\begin{array}{l}94 \\
98 \\
98\end{array}$ & $\begin{array}{l}91 \\
99\end{array}$ & $\begin{array}{r}99 \\
104\end{array}$ & $\begin{array}{r}99 \\
104\end{array}$ & $\begin{array}{r}99 \\
103\end{array}$ & $\begin{array}{l}108 \\
115\end{array}$ & $\begin{array}{l}115 \\
113\end{array}$ & $\begin{array}{l}106 \\
107\end{array}$ & \\
\hline 3 & 7 & & 64 & 76 & 89 & 92 & 93 & 98 & 97 & 101 & 110 & 114 & \\
\hline 4 & 1 & & 106 & 103 & 95 & 90 & 90 & 88 & 87 & 90 & 85 & 78 & \\
\hline 5 & 1 & & 82 & 79 & 66 & 72 & 88 & 95 & 97 & 92 & 93 & 96 & 1 \\
\hline 6 & 1 & & 87 & 87 & 85 & 78 & 70 & 85 & 96 & 95 & 93 & 92 & \\
\hline 7 & 1 & & 42 & 49 & 58 & 62 & 67 & 67 & 68 & 72 & 78 & 91 & \\
\hline 8 & 3 & & 96 & 97 & 104 & 109 & 111 & 112 & 114 & 114 & 117 & 118 & 1 \\
\hline 9 & & & 100 & 101 & 102 & 100 & 109 & 119 & 115 & 109 & 115 & 119 & \\
\hline 10 & & & 75 & 84 & 95 & 98 & 98 & 96 & 97 & 109 & 118 & 119 & \\
\hline 11 & & & 87 & 89 & 92 & 82 & 77 & 86 & 94 & 98 & 99 & 103 & \\
\hline 12 & & & 82 & 82 & 90 & 90 & 92 & 100 & 102 & 103 & 108 & 105 & \\
\hline 13 & & & 47 & 44 & 41 & 46 & 54 & 63 & 69 & 63 & 66 & 72 & \\
\hline 14 & & & 57 & 55 & 58 & 56 & 50 & 45 & 58 & 70 & 73 & 74 & \\
\hline 15 & & & 70 & 81 & 82 & 82 & 79 & 85 & 86 & 97 & 97 & 100 & \\
\hline 16 & & & 53 & 59 & 56 & 52 & 46 & 53 & 63 & 72 & 76 & 78 & \\
\hline 17 & & & 58 & 54 & 57 & 70 & 73 & 74 & 83 & 83 & 82 & 93 & \\
\hline 18 & & & 54 & 53 & 59 & 69 & 76 & 84 & 85 & 90 & 93 & 94 & \\
\hline 19 & & & 58 & 52 & 59 & 73 & 82 & 87 & 88 & 91 & 96 & 104 & 1 \\
\hline 20 & & & 72 & 75 & 76 & 73 & 70 & 59 & 62 & 56 & 56 & 54 & \\
\hline 21 & & & 65 & 64 & 66 & 66 & 58 & 55 & 67 & 80 & 88 & 92 & \\
\hline 22 & & & 78 & 73 & 71 & 69 & 67 & 65 & 63 & 56 & 54 & 66 & \\
\hline 23 & & & 81 & $\begin{array}{l}86 \\
86\end{array}$ & 84 & 77 & 79 & 年 & $\begin{array}{l}85 \\
85\end{array}$ & 8 & $\begin{array}{l}34 \\
77\end{array}$ & 73 & \\
\hline 24 & & & 42 & 48 & 51 & 51 & 56 & 54 & 55 & 91 & 109 & 117 & 1 \\
\hline 25 & & & 46 & 53 & 51 & 54 & 58 & 62 & 85 & 95 & 108 & 116 & \\
\hline 26 & & & 49 & 53 & 54 & 55 & 59 & 61 & 87 & 104 & 119 & 119 & \\
\hline 27 & & & 54 & 74 & 80 & 91 & 78 & 62 & 68 & 87 & 105 & 120 & 1 \\
\hline
\end{tabular}

In this step defined the inputloutput in neuroshell2, to define the type of each variable in my data file, have to select a type from the Variable Type selection box. This box defaults to Input and can be changed at any time to Actual or Unused. Once select a Variable Type, just click or select a range on the first row of the Define Input $\backslash$ Output grid. The cell(s) selectedwill be filled with a letter for the type selected. Input columns will appear with an I in the first row, Actual Outputs will appear with an A, and unused columns will appear blank. This method gives flexibility to experiment with different variables as inputs and outputs without having to restructure data file each time. The option to Compute Standard Deviation will allow setting the maximums and minimums to mean plus and minus $\mathrm{N}$ standard deviations. This option is handy because you can set minimum and maximum values to be narrower than the range of the actual data and clip off extreme values.As in Fig. 3.

\begin{tabular}{|c|c|c|c|c|c|c|c|c|}
\hline \multicolumn{7}{|c|}{ Define Inputs/Outputs - Hilvesourceslexe |problem 1/rejection 1.pat } & \multicolumn{2}{|c|}{\begin{tabular}{|l|l|l|}
0 & $\mathbb{1}$ & $\mathrm{X}$ \\
\end{tabular}} \\
\hline \multicolumn{9}{|c|}{ File Edit Settings Help } \\
\hline \multicolumn{9}{|c|}{ Variable Type Selection Actual Output } \\
\hline Variable Name & C1 & C2 & C3 & C4 & C5 & C6 & C7 & $\Delta$ \\
\hline Variable Type & A & $\pi$ & 1 & 1 & 1 & I & I & 1 \\
\hline Min: & 1 & 19 & 18 & 18 & 18 & 18 & 20 & 21 \\
\hline Max. & 7 & 170 & 170 & 174 & 175 & 173 & 176 & 176 \\
\hline Mean & 3.92 & 71.16 & 72.13 & 72.465 & 73.855 & 75.79 & 77.13 & 81.4 \\
\hline Std. Deviation & 1.998391 & 27.65208 & 27.87126 & 27.98526 & 28.7053 & 29.60555 & 29.73185 & 30.7 \\
\hline
\end{tabular}

Now defined what the inputs and outputs will be to our neural network, it is to build a test set of data. Before training the network, it often works better if extracted a portion of the original pattern file and use it to optimize the generalization capabilities of the net. This subset of the pattern file is called the test set. The default has been set to extract $20 \%$ of the patterns from the .PAT file in a random manner to create a test set (problemname.TST file) show fig.4. Also have the option of extracting a production file 
(problemname.PRO) from this module. The remaining patterns from the .PAT file will be assigned to the training set (problemname.TRN file). Can also extract every Nth pattern from the .PAT fileto create the .TST file (and the .PRO file) by using the second option.As in fig. 5. The rest of the patterns are assigned to the training set.

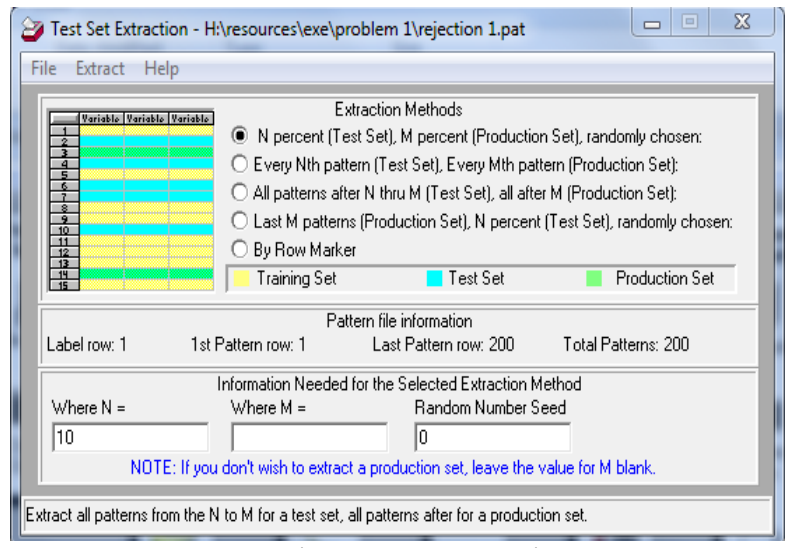

Fig. 4 Test Set Extraction

\begin{tabular}{|l|l|}
\hline Test Set Extract & \\
$\begin{array}{ll}\text { Files Extracted } \\
\text { Number of rows in training set (.trn) } \\
\text { Number of rows in test set (.tst) } \\
\text { Pattern (.pat) file was not altered. }\end{array}$ & $=180$ \\
& \\
& OK \\
\hline
\end{tabular}

Fig. 5 (trn. And tst.) Files Numbers

\section{B. System Design and train}

1) ANN Selection Model and System Design

The following discussion the fundamentals of procedures to design a new image vision based face recognition, in this research used artificial neural network to approach model detecting impression by recognize the human face expression. This following procedures applied in neuroShell2 software after created the pattern file from JAFFE data base.

To design the network that is appropriate for your problem and manipulates the network parameters. The example problem and learn about a few architectures available in NeuroShell 2. We will be choice at one architecture from each of the followingtypes: Regular feed-forward Neural Nets, Feed-forward nets with jump connections, Recurrent Neural Nets, and Ward Nets. Each type has three architectures. We will also see Kohonen Neural Net, Probabilistic Neural Nets, General Regression Neural Nets, and Polynomial (GMDH) Nets. As in fig. 6 and fig. 7.
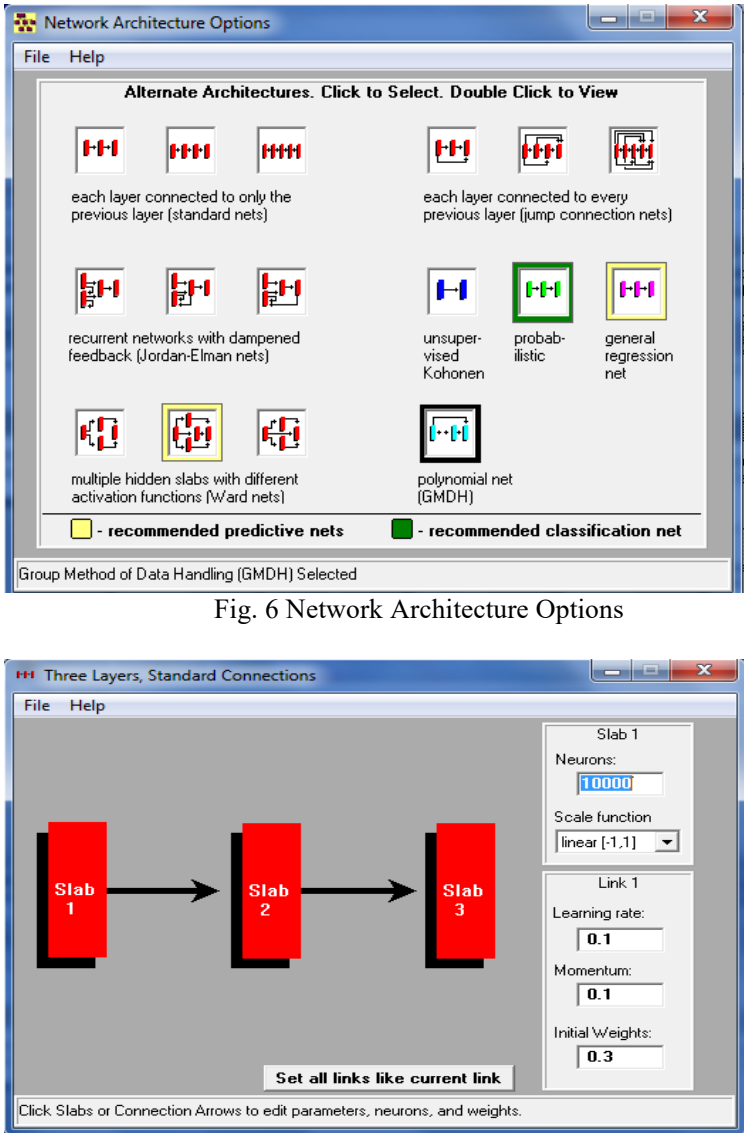

Fig. 7 3-Layers Back Propagation ANN

2) ANN System Train

Learning step to access the module that trains the neural network. The Learning screen provides features to view the training graphically and automatically save the trained network based on its performance on the training set or the test set. As in Figure 8.

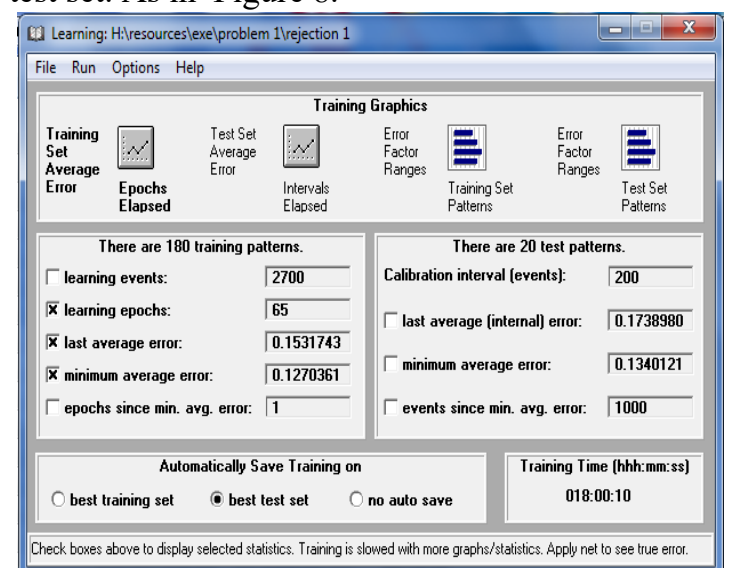

Fig. 8 ANN Learning

As in the first procedure of face impression training and test we convert the data set size from $256 * 256$ to $100 * 100$ and then converted to ASCII file through change image format to bmp. And to grd. After that saved in excel file which contain 200 patterns consist of seven face expressions. This face expression used in artificial neural network learning and expression codes.

Defined the input and output in NeuroShell2 to extract the patterns with $90 \%$ from patterns training process and $10 \%$ from patterns training set. Apply file pattern in 3-layers back propagation artificial neural network and learning patterns to 
recognize the face impression by detect the expression. The training time take long time above 65 hours.

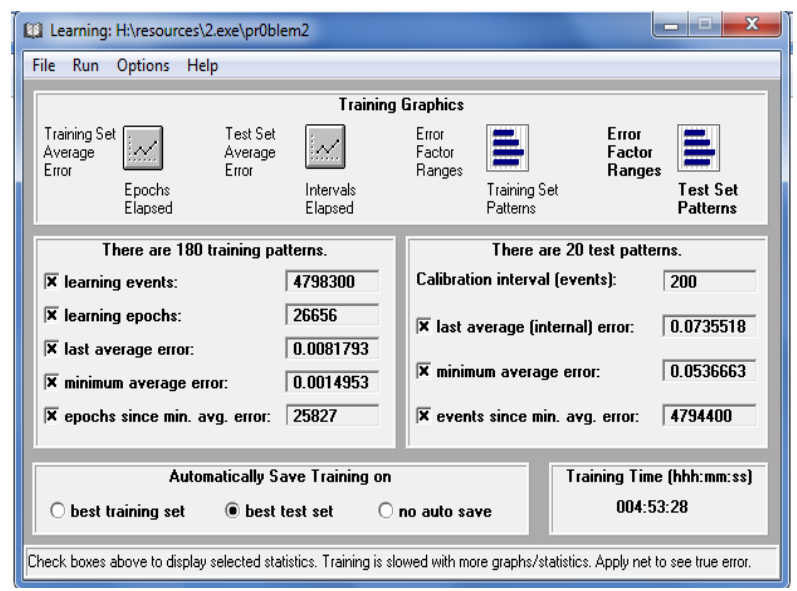

Fig. 9 ANN Test

The second procedure of impression training we resize the images to $20 * 20$ and learning network. The training time in this learning taken time less than first training time which take four hours. In the third procedure of face impression we test the network and learning by divided the all patterns to training pattern and test patterns. As in fig. 9.The training patterns percentage is $90 \%$ and test pattern percentage is $10 \%$ from all patterns.

\section{RESUlts}

After train three back propagation artificial neural network model designed and tested the results obtain as shown in (Table I).

TABLE I: THE MODEL TEST RESULTS

\begin{tabular}{lll}
\hline \hline Actual Output & $\begin{array}{l}\text { Predicted } \\
\text { Output }\end{array}$ & Error \\
\hline 7 & 6.584417 & 0.416 \\
1 & 1.239618 & 0.240 \\
2 & 1.793693 & 0.206 \\
2 & 2.539590 & 0.540 \\
2 & 2.158643 & 0.159 \\
4 & 4.332502 & 0.333 \\
4 & 4.553732 & 0.554 \\
4 & 4.066753 & 0.067 \\
3 & 3.624150 & 0.624 \\
3 & 3.511419 & 0.511 \\
5 & 5.156519 & 0.157 \\
5 & 5.371190 & 0.371 \\
5 & 5.147048 & 0.147 \\
6 & 6.246311 & 0.246 \\
6 & 6.056231 & 0.056 \\
6 & 6.337736 & 0.338 \\
7 & 5.237054 & 1.763 \\
7 & 6.276413 & 0.724 \\
1 & 1.359972 & 0.360 \\
1 & 1.494530 & 0.495 \\
1 & 1.252457 & 0.252 \\
2 & 2.078906 & 0.079 \\
2 & 1.657644 & 0.342 \\
2 & 2.523403 & 0.523 \\
4 & 3.972164 & 0.028 \\
& & \\
\hline & &
\end{tabular}

\begin{tabular}{lll}
4 & 3.823638 & 0.176 \\
4 & 4.092692 & 0.093 \\
3 & 3.295950 & 0.296 \\
3 & 3.141382 & 0.141 \\
3 & 2.984419 & 0.016 \\
5 & 5.112354 & 0.112 \\
5 & 4.131151 & 0.869 \\
5 & 5.084883 & 0.085 \\
6 & 6.074705 & 0.075 \\
6 & 5.198853 & 0.801 \\
6 & 5.934494 & 0.066 \\
1 & 1.186537 & 0.187 \\
1 & 1.000000 & 0.000 \\
1 & 1.148288 & 0.148 \\
3 & 3.345731 & 0.346 \\
3 & 3.274391 & 0.274 \\
3 & 3.276553 & 0.277 \\
5 & 5.316187 & 0.316 \\
5 & 5.340569 & 0.341 \\
5 & 5.295243 & 0.295 \\
\hline \hline Average Error & 0.321 & \\
\hline \hline
\end{tabular}

The performance of model to recognize the expressions as show in (Table II).

\begin{tabular}{|c|c|c|}
\hline Actual Impression & $\begin{array}{l}\text { Predicted } \\
\text { Impression }\end{array}$ & Performance \\
\hline Nervous & Nervous & Pass \\
\hline Happy & Happy & Pass \\
\hline Sad & Sad & Pass \\
\hline Sad & Angry & Fail \\
\hline Sad & Sad & Pass \\
\hline Surprise & Surprise & Pass \\
\hline Surprise & Disgusts & Fail \\
\hline Surprise & Surprise & Pass \\
\hline Angry & Surprise & Fail \\
\hline Angry & Surprise & Fail \\
\hline Disgusts & Disgusts & Pass \\
\hline Disgusts & Disgusts & Pass \\
\hline Disgusts & Disgusts & Pass \\
\hline Fear & Fear & Pass \\
\hline Fear & Fear & Pass \\
\hline Fear & Fear & Pass \\
\hline Nervous & Disgusts & Fail \\
\hline Nervous & Fear & Fail \\
\hline Happy & Happy & Pass \\
\hline Happy & Happy & Pass \\
\hline Happy & Happy & Pass \\
\hline Sad & Sad & Pass \\
\hline Sad & Sad & Pass \\
\hline Sad & Angry & Fail \\
\hline Surprise & Surprise & Pass \\
\hline Surprise & Surprise & Pass \\
\hline Surprise & Surprise & Pass \\
\hline Angry & Angry & Pass \\
\hline Angry & Angry & Pass \\
\hline Angry & Angry & Pass \\
\hline Disgusts & Disgusts & Pass \\
\hline
\end{tabular}




\begin{tabular}{lll} 
Disgusts & Surprise & Fail \\
Disgusts & Disgusts & Pass \\
Fear & Fear & Pass \\
Fear & Disgusts & Fail \\
Fear & Fear & Pass \\
Happy & Happy & Pass \\
Happy & Happy & Pass \\
Happy & Happy & Pass \\
Angry & Angry & Pass \\
Angry & Angry & Pass \\
Angry & Angry & Pass \\
Disgusts & Disgusts & Pass \\
Disgusts & Disgusts & Pass \\
Disgusts & Disgusts & Pass \\
\hline \hline Processed Patterns & \\
\hline \hline
\end{tabular}

\section{Where}

Actual output: represented actual code number expressions, Happy $=1, \quad \mathrm{Sad}=2, \quad$ Angry $=3, \quad$ Surprise $=4, \quad$ Disgusts $=5$, Fear $=6$, Nervous $=7$.

Predicted output: output of network after learning patterns. Error: network errors.

Actual impression: rounded actual output (Colum b)

Predicted Impression: rounded predicted output (Colum c)

Performance: if actual impression $=$ predicted impression the performance is pass otherwise is fail.

Processed Patterns: percentage of patterns which running in network.

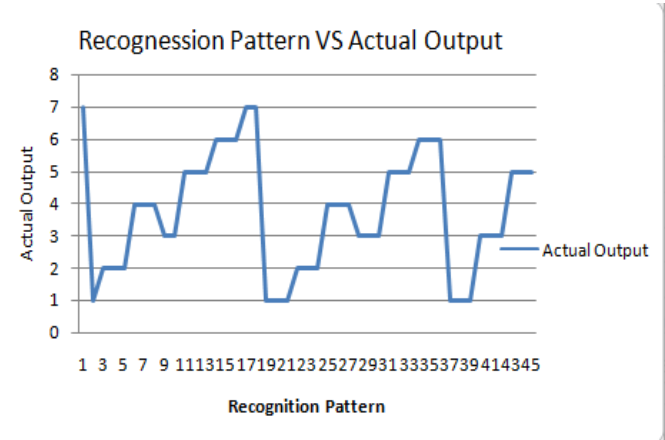

Fig. 10 Actual Outputs

Recognition Pattern VS Predicted Output

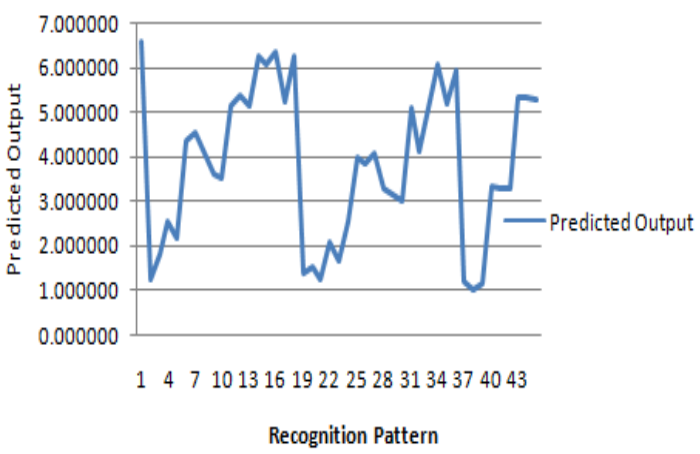

Fig. 11 Predicted Outputs

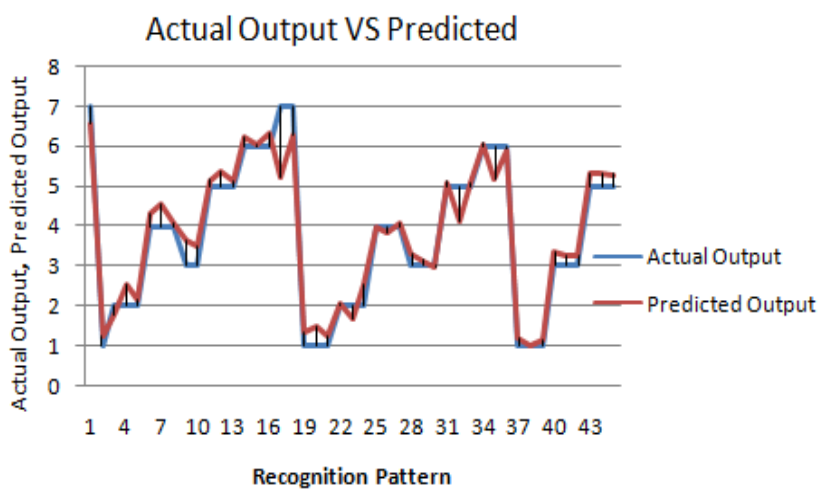

Fig. 12 Actual Outputs VS Predicted Outputs

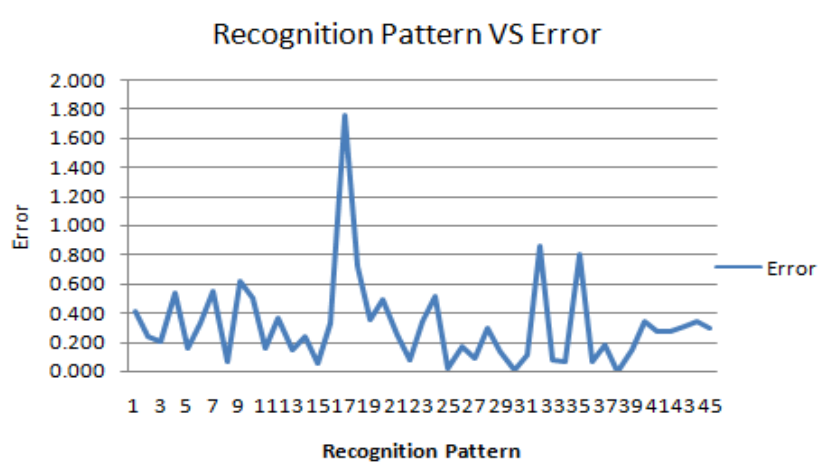

Fig. 13 Network Recognition Error

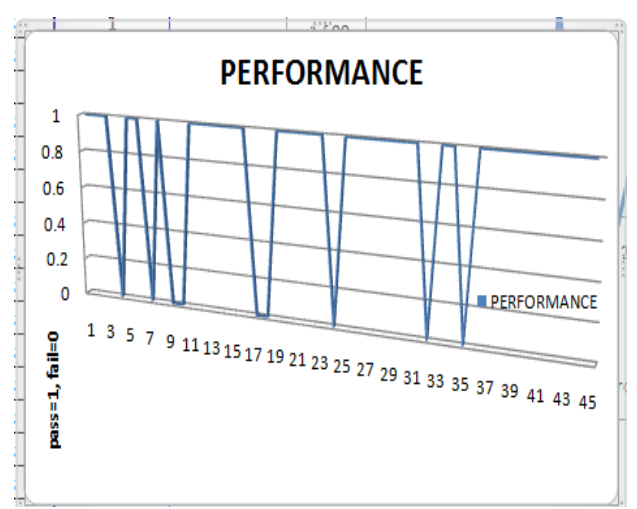

Fig. 14 Network Recognition Performance

\section{CONCLUSION}

When running the first experiment of neural network training, the time taken for the implementation process was long and slow response, so the patterns were reprocessed by adjusting the image size from $100 * 100$ to $20 * 20$. The abnormal patterns were eliminated. The network response was faster. Neurophysiology of the extent to which the impression is obtained through the expression of the human face by comparing the actual value with the expected value after neural network training.

The test group consisted of 45 patterns representing $25 \%$ of the total patterns and the performance was assessed in Table. I. Give a pass if the actual values correspond to the expected value of the grid and give a failure if there is no match.

Fig. 10 shows the actual values in which the expressions are represented and takes the values from 1 to 7 . Fig. 11 shows the expected values of the neural network after training on the set of patterns. The fig. 12 illustrates the relationship between actual output and predicted output. Fig. 13 shows 
neural network recognition error. And fig. 14 show recognition performance.

Through the training of the network and analysis of the results were the percentage of impression recognition $80 \%$ and the percentage of failure to recognition the impression $20 \%$.

\section{REFERENCES}

[1] Virgina Espinosa Duro, Face Recognition By Means of Advanced Contributions in Machine Learning,Ph.D. thesis, University of Politècnica de Catalunya,Espan.:May, 2013.

[2] Grossberg, S., Adaptive pattern classification and universal recoding 1976.

[3] McCulloch, W.S., Pitts, W., A logical calculus of the ideas immanent 1943.

[4] Hebb, D., The Organization of Behavior. Wiley, New York1949.

[5] FarshadGhahramani ,Face Recognition: An Engineering Approach, San Jose State University 2015.

[6] T. F. Cootes, K. Walker and C. J. Taylor Grenoble, "View based active appearance models, "IEEE International Conference on Automatic Face and Gesture Recognition, France, pp. 227-23 Mar 2000 .

[7] B. Moghaddam, T. Jebara and A. Pentlandvol, Baysian face recognition, in Pattern Recognition, Nov 2000.

[8] P. Penev and J. Atick, vol, Local feature analysis: a general statistical theory for object representation, in Network: Computation in Neural Systems Oct 1996 